\title{
siam.
}

Minimal Representations for Translation-Invariant Set Mappings by Mathematical Morphology Author(s): Gerald Jean Francis Banon and Junior Barrera

Source: SIAM Journal on Applied Mathematics, Vol. 51, No. 6 (Dec., 1991), pp. 1782-1798

Published by: Society for Industrial and Applied Mathematics

Stable URL: http://www.jstor.org/stable/2102371

Accessed: 02/04/2013 13:46

Your use of the JSTOR archive indicates your acceptance of the Terms \& Conditions of Use, available at http://www.jstor.org/page/info/about/policies/terms.jsp

JSTOR is a not-for-profit service that helps scholars, researchers, and students discover, use, and build upon a wide range of content in a trusted digital archive. We use information technology and tools to increase productivity and facilitate new forms of scholarship. For more information about JSTOR, please contact support@jstor.org. 


\title{
MINIMAL REPRESENTATIONS FOR TRANSLATION-INVARIANT SET MAPPINGS BY MATHEMATICAL MORPHOLOGY*
}

\author{
GERALD JEAN FRANCIS BANON† AND JUNIOR BARRERA $\dagger$
}

\begin{abstract}
In his 1975 book, Matheron introduced a pair of dual representations, written in terms of erosions and dilations, for increasing translation invariant set mappings, using the concept of a kernel. Based on hit-miss topology, Maragos, in his 1985 Ph.D. thesis, has given sufficient conditions under which the increasing mappings have minimal representations. In this paper, a pair of dual representations for translation-invariant set mappings (not necessarily increasing) is presented. It is shown that under the same sufficient conditions such mappings have minimal representations. Actually, the representations of Matheron and Maragos are special cases of the proposed ones. Finally, some examples are given to illustrate the theory.
\end{abstract}

Key words. mathematical morphology, dilation, erosion, translation-invariant mapping, lattice, closed interval, convex set, kernel, basis, hit-miss topology, edge extraction, shape recognition, image processing

AMS(MOS) subject classifications. 06A23, 68U10, 93A10, 94A12, 68T10

1. Introduction. Mathematical morphology, introduced in the 1960 s by Matheron and Serra at "École des Mines de Paris," appears to play more and more an important role in the field of image processing (Serra (1982); Haralick, Sternberg, and Zhuang (1987); Giardina and Dougherty (1988)).

In mathematical morphology, a shape or image (binary image) is seen as a subset of a Euclidean space and any image processing scheme is viewed as a mapping between collections of subsets. The key idea of the theory is the representation of mappings by two elementary ones: erosions and dilations. Each erosion and dilation is characterized by a subset called a structuring element that acts as a probe in the analysis of a given shape or image.

A natural question that arises is: what class of mappings can be studied by mathematical morphology? Or, in other words, what class of mappings can be built by erosions and dilations? A first interesting result to this problem was given by Matheron.

In his pioneering work, Matheron (1975) has shown that the set of translationinvariant (t.i.) set mappings, whose domain is a collection $\mathscr{A}$ (closed under translation) of subsets of a Euclidean space, is isomorphic to the set of all subcollections of $\mathscr{A}$. In order to clarify this point, Matheron introduced the concept of a kernel of a t.i. set mapping $\psi$, which is defined as the subcollection of subsets in $\mathscr{A}$, whose transforms by $\psi$ contain the null element of the Euclidean space. Hence, for example, the kernel of an erosion by a structuring element $A$ is the subcollection of all subsets in $\mathscr{A}$ containing $A$.

By using the fact that the kernel of an increasing t.i. set mapping is a dual ideal (Matheron says that the kernel is U-hereditary), Matheron (1975) has shown that any increasing t.i. set mapping $\psi$ can be represented as the supremum of erosions by structuring elements in the kernel of $\psi$.

${ }^{*}$ Received by the editors October 31, 1988, accepted for publication (in revised form) October 17, 1990. This work was supported by the FAPESP ("Fundação de Amparo à Pesquisa do Estado de São Paulo") under contract 87/2478-9 and SID INFORMÁTICA S/A through the ESTRA ("Estações de Trabalho Avonçadas") project.

† Instituto Nacional de Pesquisas Espaciais (INPE), Image Processing Department, Av. dos Astronautas, 1758, Caixa Postal 515, 12201, São José dos Campos, Brazil. 
The first objective of this paper is to extend Matheron's results to the case of nonnecessarily increasing mappings. The kernel of a t.i. set mapping is no longer a dual ideal and the proposed solution consists in using the concept of closed interval of subsets in $\mathscr{A}$. We first identify the so-called sup-generating mappings characterized by a pair of structuring elements (instead of one for the case of erosion), whose kernel is a closed interval. Then we show that any set mapping $\psi$ can be represented as the supremum of sup-generating mappings, whose structuring elements are the extremities of the closed intervals included in the kernel of $\psi$.

This result is important because a sup-generating mapping appears to be the infimum of an erosion and the composition of an erosion and a complementation, that is, it shows that erosions and complementation are prototypes of any t.i. set mapping.

As in the increasing case, it is possible to derive a dual representation in terms of dilations.

Because the above representation is usually redundant, the second objective of this paper is to present some minimal representation results similar to the ones given by Maragos (1985), (1989) and Dougherty and Giardina (1986) for increasing t.i. set mappings, and to indicate the close relationship between these results and some of the switching theory related to the simplification techniques for Boolean functions.

In $\S 2$, two dual representation theorems are given for a nonnecessarily increasing t.i. set mapping. In $\S 3$, the representations by a supremum and an infimum are specialized for the so-called inf-separable and sup-separable mappings, which are mappings that can be decomposed by a pair of increasing and decreasing ones. In particular, the inf-separable mappings are the ones that have a convex kernel. Section 4 contains the definition of t.i. set mapping basis as well as sufficient conditions, expressed in terms of semicontinuity in the hit-miss topology, under which a t.i. set mapping has minimal representations. Finally, in $\S 5$, some simple examples are given to illustrate the theory.

\section{Representation theorems.}

2.1. Representation by a supremum. We first introduce some definitions.

Let $\mathscr{A}$ be a nonempty collection of subsets of a nonempty set $E$, that is, $\mathscr{A} \subset \mathscr{P}(E)$. The set $\Psi$ of all the mappings $\psi$ from $\mathscr{A}$ to $\mathscr{P}(E)$ inherits the complete lattice structure of $(\mathscr{P}(E), \subset)$ by setting

$$
\psi_{1}<\psi_{2} \quad \text { iff } \psi_{1}(X) \subset \psi_{2}(X)(X \in \mathscr{A}) .
$$

The supremum and infimum of a subset $\Theta$ of the complete lattice $(\Psi,<)$ verify

$$
(\bigvee \Theta)(X)=\bigcup\{\theta(X): \theta \in \Theta\} \quad(X \in \mathscr{A})
$$

and

$$
(\bigwedge \Theta)(X)=\cap\{\theta(X): \theta \in \Theta\} \quad(X \in \mathscr{A}),
$$

respectively. Moreover, $(\Psi,<)$ is a distributive lattice.

In this paper, we focus our attention on a subset of $\Psi$ that is commonly used in image processing, namely, the subset of translation-invariant mappings. For this purpose, $E$ is assumed to be an Abelian group with respect to a binary operation denoted by + . The zero element of $(E,+)$ is denoted by 0 .

For any $h \in E$ and $X \subset E$, the set $X_{h}=\{y \in E: y=x+h, x \in X\}$ is called the translate of $X$ by $h$. In particular, $X_{\mathrm{o}}=X$. We denote by $-x$ the opposite of any $x$ in $E$.

From now on, the domain $\mathscr{A}$ is supposed to be closed under translation, that is, for any $h \in E$ and $X \in \mathscr{A}, X_{h} \in \mathscr{A}$. Let $\Phi_{\mathscr{A}}$ or simply $\Phi$ be the subset of $\Psi$ of all 
translation-invariant (t.i.) mappings $\psi$ from $\mathscr{A}$ to $\mathscr{P}(E)$, that is, the mappings such that

$$
\psi\left(X_{h}\right)=(\psi(X))_{h} \quad(X \in \mathscr{A}, \boldsymbol{h} \in E) .
$$

We can verify that the partially ordered set $(\Phi,<)$ is a complete sublattice of $(\Psi,<)$. An important notion related to t.i. mappings is that of a kernel, introduced by Matheron (1975). The kernel $\mathscr{K}(\psi)$ of a t.i. mapping $\psi$ from $\mathscr{A}$ to $\mathscr{P}(E)$ is the subcollection of $\mathscr{A}$ defined by

$$
\mathscr{K}(\psi)=\{X \in \mathscr{A}: 0 \in \psi(X)\} .
$$

We now introduce a subset of t.i. mappings from which it will be possible to represent any mapping in $\Phi$ by a supremum. This subset is formed by all the so-called sup-generating mappings $\cdot \otimes(A, B)$ defined by

$$
X \otimes(A, B)=\left\{x \in E: A_{x} \subset X \subset B_{x}\right\} \quad(X \in \mathscr{A})
$$

and characterized by two subsets $A$ and $B$ in $\mathscr{A}$ and such that $A \subset B$.

As will be seen below, the representation by a supremum will be derived directly from the notion of a closed interval and a very simple lemma.

Let $(P, \leqq)$ denote a partially ordered set. Given $\boldsymbol{a} \leqq \boldsymbol{b}$ in $\boldsymbol{P}$, the subset of all elements $x \in P$ that satisfy $a \leqq x \leqq b$ is called a closed interval of $P$ and denoted by $[a, b]_{P}$ or simply by $[a, b]$ (Birkhoff $(1967$, p. 7$)$ ). The elements $a$ and $b$ are the extremities of $[a, b]$.

Lemma 2.1. Any subset $S$ of $(P, \leqq)$ can be properly covered by the union of the closed intervals of $P$ contained in $S$, that is,

$$
S=\bigcup\{[a, b]:[a, b] \subset S\} .
$$

Proof. By construction, we have

$$
S \supset \bigcup\{[a, b]:[a, b] \subset S\} .
$$

On the other hand, for any $x \in S$ we have $[x, x] \subset S$, but $x \in[x, x]$; therefore

$$
x \in \bigcup\{[a, b]:[a, b] \subset S\}
$$

and consequently

$$
S \subset \cup\{[a, b]:[a, b] \subset S\} .
$$

We are now ready to state and prove the main result of this paper.

THEOREM 2.1 (Translation-invariant mapping representation by a supremum). Let $\psi$ be a t.i. mapping and $\mathscr{K}(\psi)$ its kernel; then

$$
\psi=\bigvee\{\cdot \otimes(A, B):[A, B] \subset \mathscr{K}(\psi)\}
$$

In other words,

$$
\psi(X)=\bigcup\{X \otimes(A, B):[A, B] \subset \mathscr{K}(\psi)\} \quad(X \in \mathscr{A}) .
$$

Proof. Let $\psi$ be any t.i. mapping. By using Lemma 2.1, the subcollection $\mathscr{K}(\psi)$ of the partially ordered collection $(\mathscr{A}, \subset)$ can be written

$$
\mathscr{K}(\psi)=\bigcup\{[A, B]:[A, B] \subset \mathscr{K}(\psi)\} .
$$

On the other hand, we have

$$
\mathscr{K}(\cdot \otimes(A, B))=[A, B] .
$$


Furthermore, it is known (Matheron (1975, Chap. 8); Banon and Barrera (1990, Chap. 2) for a detailed proof) that the mapping $\psi \mapsto \mathscr{K}(\psi)$ is a lattice-isomorphism from $\left(\Phi_{\mathscr{A}},<\right)$ to $(\mathscr{P}(\mathscr{A}), \subset)$; therefore, the result follows by applying the corresponding inverse mapping to both sides of

$$
\mathscr{K}(\psi)=\bigcup\{\mathscr{K}(\cdot \otimes(A, B)):[A, B] \subset \mathscr{K}(\psi)\} .
$$

It is interesting to note that the sup-generating mapping $\bullet(A, B)$ is actually the infimum (this is the reason for using the symbol $\otimes$ ) of an erosion and an antidilation. This can be seen by writing

$$
X \otimes(A, B)=\left\{x \in E: A_{x} \subset X\right\} \cap\left\{x \in E: B_{x}^{c} \subset X^{c}\right\} .
$$

The first set on the right-hand side is the erosion of $X$ by $A$ and the second is the erosion of $X^{c}$ (the complementary set of $X$ ) by $B^{c}$, that is, the result of an antidilation of $X$ (Serra (1987)). In terms of the Minkowski subtraction $\ominus \ominus \cdot($ Hadwiger (1950); Sternberg (1982)), we have

$$
\cdot\left(\Delta(A, B)=(\cdot \ominus A) \wedge\left(\cdot{ }^{c} \ominus B^{c}\right)\right.
$$

In terms of the Serra hit-miss transformation $\otimes(A, B)$ (Serra (1982)), we have

$$
\cdot \otimes(A, B)=\cdot \otimes\left(A, B^{c}\right) \text {. }
$$

2.2. Representation by an infimum. Once the representation by a supremum has been established, the easiest way to introduce the representation by an infimum is by using the duality principle.

Let $\mathscr{A}^{*}=\left\{X \subset E: X^{c} \in \mathscr{A}\right\}$ be the collection of the complementary sets of sets in $\mathscr{A}$. The mapping from $\Phi_{\mathscr{A}}$ to $\Phi_{\mathscr{A}^{*}}$ defined by $\psi \mapsto \psi^{*}$, where the dual mapping $\psi^{*}$ (from $\mathscr{A}^{*}$ to $\mathscr{P}(E)$ ) is defined by

$$
\psi^{*}(X)=\left(\psi\left(X^{c}\right)\right)^{c} \quad\left(X \in \mathscr{A}^{*}\right),
$$

is decreasing two-sided (i.e., $\psi_{1}<\psi_{2}$ if and only if $\psi_{2}^{*}<\psi_{1}^{*}$ ) and bijective, in other words, the mapping $\psi \mapsto \psi^{*}$ is a dual lattice-isomorphism from $\left(\Phi_{\mathscr{A}},<\right)$ to $\left(\Phi_{\mathscr{A}^{*}},<\right)$.

Let $\check{A}=\{x \in E:-x \in A\}$ be the symmetrical set of a set $A \subset E$. We now introduce a subset of t.i. mappings in $\Phi_{\mathscr{A}}$ from which it will be possible to represent any mapping in $\Phi_{\mathscr{A}}$ by an infimum. This subset is formed by all the so-called inf-generating mapping - (1) $(A, B)=(\cdot \otimes(\check{A}, \check{B}))^{*}$ characterized by two subsets $A$ and $B$ in $\mathscr{A}^{*}$ and such that $A \subset B$.

By definition of dual mapping, we have

$$
X \otimes(A, B)=\left\{x \in E: X \cap \check{A}_{x} \neq \varnothing \text { or } X \cup \check{B}_{x} \neq E\right\} \quad(X \in \mathscr{A}) .
$$

TheOREM 2.2 (Translation-invariant mapping representation by an infimum). Let $\psi$ be a t.i. mapping from $\mathscr{A}$ to $\mathscr{P}(E)$ and $\mathscr{K}\left(\psi^{*}\right)$ the kernel of its dual; then

$$
\psi=\bigwedge\left\{\cdot \otimes(\check{A}, \check{B}):[A, B]_{\mathscr{A}^{*}} \subset \mathscr{K}\left(\psi^{*}\right)\right\} .
$$

In other words,

$$
\psi(X)=\bigcap\left\{X \oplus(\check{A}, \check{B}):[A, B]_{\mathscr{A}^{*}} \subset \mathscr{K}\left(\psi^{*}\right)\right\} \quad(X \in \mathscr{A}) .
$$

Proof. By Theorem 2.1,

$$
\psi^{*}=\bigvee\left\{\cdot \otimes(A, B):[A, B]_{\mathscr{A}} \subset \mathscr{K}\left(\psi^{*}\right)\right\}
$$


By applying the inverse of the dual lattice-isomorphism $\psi \mapsto \psi^{*}$ to both sides, we have

$$
\psi=\bigwedge\left\{(\cdot \otimes(A, B))^{*}:[A, B]_{\mathscr{A}^{*}} \subset \mathscr{K}\left(\psi^{*}\right)\right\} .
$$

The result then follows from the definition of $\cdot(B)(A, B)$.

It is interesting to note that the inf-generating mapping $\cdot(\mathbb{v}(A, B)$ is actually the supremum (this is the reason for using the symbol $\otimes$ ) of a dilation and an anti-erosion. This can be seen by writing

$$
X \otimes(A, B)=\left\{x \in E: X \cap \check{A}_{x} \neq \varnothing\right\} \cup\left\{x \in E: X^{c} \cap \check{B}_{x}^{c} \neq \varnothing\right\} .
$$

Following Sternberg's definition (Matheron and Serra's definition is slightly different), the first set on the right-hand side is the dilation of $X$ by $A$ and the second one is the dilation of $X^{c}$ by $B^{c}$, that is, the result of an anti-erosion of $X$ (Serra (1987)). In terms of the Minkowski addition $\oplus \cdot$ (Minkowski (1903); Hadwiger (1950); Sternberg (1982)), we have

$$
\cdot(\nabla)(A, B)=(\cdot \oplus A) \vee\left(\cdot{ }^{c} \oplus B^{c}\right) \text {. }
$$

3. Increasing, decreasing, inf-separable, and sup-separable mappings. The objective of this section is to specialize the representation theorems of the previous section to the cases of increasing, decreasing, inf-separable, and sup-separable t.i. mappings. In the former case, we will obtain Matheron's representation theorem.

A mapping $\psi$ from $\mathscr{A}$ to $\mathscr{P}(E)$ is said to be:

increasing if and only if for any $X$ and $Z \in \mathscr{A}, X \subset Z$ implies that $\psi(X) \subset \psi(Z)$; decreasing if and only if for any $Y$ and $Z \in \mathscr{A}, Z \subset Y$ implies that $\psi(Y) \subset \psi(Z)$.

Erosion and dilation are examples of increasing mappings. Anti-erosion and antidilation are examples of decreasing mappings.

A mapping $\psi$ from $\mathscr{A}$ to $\mathscr{P}(E)$ is said to be:

inf-separable if and only if for any $X, Y$ and $Z \in \mathscr{A}, X \subset Z \subset Y$ implies that $\psi(X) \cap \psi(Y) \subset \psi(Z)$;

sup-separable if and only if for any $X, Y$ and $Z \in \mathscr{A}, X \subset Z \subset Y$ implies that $\psi(Z) \subset \psi(X) \cup \psi(Y)$.

From these definitions, any increasing and decreasing mappings are inf-separable and sup-separable mappings:

$X \subset Z \subset Y$ implies that

$$
\psi(X) \cap \psi(Y)=\psi(X) \subset \psi(Z) \subset \psi(Y)=\psi(X) \cup \psi(Y)
$$

if $\psi$ is increasing; and

$$
\psi(X) \cap \psi(Y)=\psi(Y) \subset \psi(Z) \subset \psi(X)=\psi(X) \cup \psi(Y)
$$

if $\psi$ is decreasing.

It is already known (Matheron $(1975$, p. 219)) that a t.i. mapping is increasing if and only if its kernel $\mathscr{K}(\psi)$ is $U$-hereditary, that is, a dual ideal of $(\mathscr{A}, \subset): X \in \mathscr{K}(\psi)$, $Z \in \mathscr{A}$, and $X \subset Z$ imply that $Z \in \mathscr{K}(\psi)$. In the same way, a t.i. mapping $\psi$ is decreasing if and only if its kernel $\mathscr{K}(\psi)$ is $\cap$-hereditary, that is, an ideal of $(\mathscr{A}, \subset): Y \in \mathscr{K}(\psi)$, $Z \in \mathscr{A}$, and $Z \subset Y$ imply that $Z \in \mathscr{K}(\psi)$.

Before presenting an interesting kernel property for inf-separable mappings, we recall the definition of a convex subset. A convex subset of a partially ordered set $(P, \leqq)$ is a subset that contains the closed interval $[a, b]$ whenever it contains $a$ and $b, a \leqq b$ (Birkhoff, (1967, p. 7)). 
Property 3.1. If $\psi$ is an inf-separable t.i. mapping, then its kernel $\mathscr{K}(\psi)$ is a convex subset of $(\mathscr{A}, \subset)$.

Proof. Let $X$ and $Y \in \mathscr{K}(\psi)$, then $o \in \psi(X)$ and $\psi(Y)$. Let $Z \in \mathscr{A}$ and $X \subset Z \subset Y$; then by the inf-separable mapping definition, $o \in \psi(Z)$, that is, $Z \in \mathscr{K}(\psi)$ and $[X, Y] \subset$ $\mathscr{K}(\psi)$.

Inf-separable and sup-separable mappings are dual concepts and are closely related to increasing and decreasing mappings as shown in the following corollary, which is a specialized version of the representation theorems of the previous section.

COROllary 3.1. Let $\psi$ be a t.i. mapping, and let $\mathscr{K}(\psi)$ be its kernel; then

(1) $\quad \psi=(\bigvee\{\cdot \ominus A: A \in \mathscr{K}(\psi)\}) \wedge\left(\bigvee\left\{\cdot{ }^{c} \ominus B^{c}: B \in \mathscr{K}(\psi)\right\}\right)$ if $\psi$ is inf-separable,

(2) $\quad \psi=\left(\bigwedge\left\{\cdot \oplus \check{A}: A \in \mathscr{K}\left(\psi^{*}\right)\right\}\right) \vee\left(\bigwedge\left\{\cdot^{c} \oplus \check{B}^{c}: B \in \mathscr{K}\left(\psi^{*}\right)\right\}\right)$ if $\psi$ is sup-separable,

(3) $\quad \psi=\bigvee\{\cdot \ominus A: A \in \mathscr{K}(\psi)\}=\bigwedge\left\{\cdot \oplus \check{A}: A \in \mathscr{K}\left(\psi^{*}\right)\right\}$ if $\psi$ is increasing,

(4) $\quad \psi=\bigvee\left\{\cdot{ }^{c} \ominus B^{c}: B \in \mathscr{K}(\psi)\right\}=\bigwedge\left\{\cdot{ }^{c} \oplus \check{B}^{c}: B \in \mathscr{K}\left(\psi^{*}\right)\right\}$ if $\psi$ is decreasing.

Proof. (1) The inf-separable case. By Property 3.1, $\mathscr{K}(\psi)$ is convex; hence, the condition $[A, B] \subset \mathscr{K}(\psi)$ is equivalent to $(A, B \in \mathscr{K}(\psi)$ and $A \subset B)$. On the other hand, - $ه(A, B)$ is the least t.i. mapping whenever $A$ is not included in $B$; therefore, by using Theorem 2.1,

$$
\psi=\bigvee\{\cdot \otimes(A, B): A, B \in \mathscr{K}(\psi)\}
$$

The result follows by writing $\bullet(A, B)$ as the infimum of an erosion and an antidilation and by distributivity of the sublattice $(\Phi,<)$.

(2) The sup-separable case. The result follows by using Theorem 2.2 and the arguments of (1) since $\psi^{*}$ is inf-separable.

(3) The increasing case. The result follows from the inf-separable case if it can be proved that

$$
\bigvee\{\cdot \ominus A: A \in \mathscr{K}(\psi)\}<\bigvee\left\{\cdot{ }^{c} \ominus B^{c}: B \in \mathscr{K}(\psi)\right\}
$$

For any $X \in \mathscr{A}$ and $A \in \mathscr{K}(\psi)$, let $x \in X \ominus A$ or, equivalently, $A_{x} \subset X$ and let $Y=X_{-x}$; then $A \subset Y$. By $U$-hereditary of $\mathscr{K}(\psi), Y \in \mathscr{K}(\psi)$, but $Y=X_{-x}$ implies, by erosion definition, that $x \in X^{c} \ominus Y^{c}$; therefore, $x \in \bigcup\left\{X^{c} \ominus B^{c}: B \in \mathscr{K}(\psi)\right\}$. The representation by an infimum follows by duality.

(4) The decreasing case. The result follows by similar arguments.

By making $\mathscr{A}=\mathscr{A}^{*}=\mathscr{P}(E)$, part (3) of Corollary 3.1 is Matheron's representation theorem (Matheron (1975)).

The terms inf-separable mapping and sup-separable mapping have been chosen because, as shown in parts (1) and (2) of Corollary 3.1, such mappings can be written, respectively, as an infimum and a supremum of two mappings: an increasing and a decreasing one. For inf-separable mappings the increasing part is a supremum of erosions, while the decreasing part is a supremum of antidilations. For the supseparable mappings the increasing part is an infimum of dilations, whereas the decreasing part is an infimum of anti-erosions.

Moreover, we can observe that the infimum (respectively, supremum) of an increasing and a decreasing mapping is an inf-separable (Matheron (1989)) (respectively, sup-separable) mapping. Hence, from the above, the statements:

(1) $\psi$ is inf-separable;

(2) $\mathscr{K}(\psi)$ is convex;

(3) $\psi=\psi_{1} \wedge \psi_{2}$ with $\psi_{1}$ increasing and $\psi_{2}$ decreasing, are actually equivalent.

As well, by duality principle, the statements:

(1) $\psi$ is sup-separable;

(2) $\psi=\psi_{1} \vee \psi_{2}$ with $\psi_{1}$ increasing and $\psi_{2}$ decreasing, are equivalent. 
In particular, the sup-generating mapping $\cdot \otimes(A, B)$ and inf-generating mapping - (1) $(A, B)$ of the previous section are examples of, respectively, inf-separable and sup-separable mappings.

\section{Minimal representation theorems.}

4.1. Algebraic aspects. The representation theorems of $\S 2$ may lead to redundant representations for most t.i. mappings in the sense that a smaller family of generating mappings may be used.

In the case of the representation by a supremum, for example, this can be seen as follows. If $[A, B] \subset\left[A^{\prime}, B^{\prime}\right]$, then $\otimes(\mathrm{A}, \mathrm{B})<\cdot \otimes\left(\mathrm{A}^{\prime}, \mathrm{B}^{\prime}\right)$. Therefore, in the representation by a supremum for a mapping $\psi$, if the above closed intervals $[A, B]$ and $\left[A^{\prime}, B^{\prime}\right]$ are contained in $\mathscr{K}(\psi)$, the sup-generating mapping $\bullet(A, B)$ will be redundant.

In order to derive minimal representations for a t.i. mapping $\psi$ from $\mathscr{A}$ to $\mathscr{P}(E)$, we now introduce two definitions.

The set $\mathbf{B}(\psi)$ of all the maximal closed intervals contained in $\mathscr{K}(\psi)$ is called the basis of $\psi$. A closed interval contained in $\mathscr{K}(\psi)$ is maximal if no other closed interval contained in $\mathscr{K}(\psi)$ properly contains it. This definition of basis differs from the ones of Maragos (1985) and Dougherty and Giardina (1986) who have defined a similar notion for increasing mappings.

The set $\mathbf{B}$ of closed intervals contained in $\mathscr{K}(\psi)$ is said to satisfy the representation condition for $\psi$ if and only if for any closed interval contained in $\mathscr{K}(\psi)$ there exists a closed interval in B that contains it.

THEOREM 4.1 (Minimal representation by a supremum). Let $\psi$ be a t.i. mapping, let $\mathscr{K}(\psi)$ be its kernel, and let $\mathbf{B}$ be a set of closed intervals contained in $\mathscr{K}(\psi)$ satisfying the representation condition for $\psi$. Then

(1) $\psi=\bigvee\{\cdot \otimes(A, B):[A, B] \in \mathbf{B}\}$

(2) Furthermore, if $\mathbf{B}(\psi)$, its basis, satisfies the representation condition for $\psi$, then

$$
\mathbf{B}(\psi) \subset \mathbf{B}, \quad \psi=\bigvee\{\cdot \otimes(A, B):[A, B] \in \mathbf{B}(\psi)\}
$$

and $\psi$ is said to have a minimal representation by a supremum.

Proof. (1) B being a set of closed intervals contained in $\mathscr{K}(\psi)$, we have

$$
\bigvee\{\cdot \otimes(A, B):[A, B] \in \mathbf{B}\}<\bigvee\{\cdot \otimes(A, B):[A, B] \subset \mathscr{K}(\psi)\}
$$

On the other hand, for any $[A, B] \subset \mathscr{K}(\psi)$ let $\left[A^{\prime}, B^{\prime}\right]$ be a closed interval in $\mathbf{B}$ such that $[A, B] \subset\left[A^{\prime}, B^{\prime}\right]$; then

$$
\bigvee\{\cdot \otimes(A, B):[A, B] \subset \mathscr{K}(\psi)\}<\bigvee\left\{\cdot \otimes\left(A^{\prime}, B^{\prime}\right):[A, B] \subset \mathscr{K}(\psi)\right\},
$$

by expanding to all the closed interval belonging to $\mathbf{B}$,

$$
<\bigvee\left\{\cdot\left(A^{\prime}, B^{\prime}\right):\left[A^{\prime}, B^{\prime}\right] \in \mathbf{B}\right\}
$$

That is, by antisymmetry of the partial order $<$,

$$
\bigvee\{\cdot(A, B):[A, B] \subset \mathscr{K}(\psi)\}=\bigvee\{\cdot \otimes(A, B):[A, B] \in \mathbf{B}\}
$$

and the result follows by using Theorem 2.1.

(2) $\mathbf{B}(\psi)$ is contained in any $\mathbf{B}$ satisfying the representation condition for $\psi$ since, otherwise, for any closed interval in $\mathbf{B}(\psi)$ and not in $\mathbf{B}$ there would exist another closed interval in $\mathbf{B}$ that contains it, that is, $\mathbf{B}(\psi)$ would not be the basis of $\psi$. 
In Theorem 4.1, we use the expression "minimal representation" because if $\mathbf{B}(\psi)$ is one of the sets $\mathbf{B}$ satisfying the representation condition, then $\mathbf{B}(\psi)$ is the least set among such $\mathbf{B}$ that can be used to represent $\psi$ by a supremum.

Actually, the representation Theorem 4.1 may still lead to a redundant representation since the union of two or more closed intervals of $\mathbf{B}(\psi)$ may contain a different closed interval of $\mathbf{B}(\psi)$ that could be discarded in the representation of $\psi$ by a supremum.

In the case of a finite set $E$ of $n$ elements, we can uniquely associate to each t.i. mapping $\psi$ from $\mathscr{P}(E)$ to $\mathscr{P}(E)$ a Boolean function $f$ of $n$ variables which takes the true value whenever the input combination corresponds to a subset $X$ satisfying the condition $0 \in \psi(X)$. In the standard representation of $f$ by a sum of minterm, each minterm corresponds to an element of the kernel of $\psi$. The usual simplification techniques for Boolean functions of the switching theory (Hill and Peterson (1974)) can be applied to simplify the representation of $\psi$ by a supremum. The determination of the so-called prime implicants of the Boolean function $f$ by the Quine-McCluskey method leads exactly to the basis of $\psi$, since the prime implicants of $f$ corresponds to the maximal closed intervals contained in $\mathscr{K}(\psi)$. Moreover, in a minimal sum-ofproduct expression of $f$, obtained by selecting an optimum set of prime implicants, as proposed by Quine and McCluskey, the products correspond to maximal closed intervals contained in $\mathscr{K}(\psi)$ which still properly cover $\mathscr{K}(\psi)$. By using all these maximal closed intervals, a representation of $\psi$ by a supremum can be achieved that is identical or even simpler than the minimal representation given in Theorem 4.1.

The dual form of the minimal representation by a supremum is now presented.

THEOREM 4.2 (Minimal representation by an infimum). Let $\psi$ be a t.i. mapping, let $\mathscr{K}\left(\psi^{*}\right)$ be the kernel of its dual, and let $\mathbf{B}$ be a set of closed intervals contained in $\mathscr{K}\left(\psi^{*}\right)$ satisfying the representation condition for $\psi^{*}$. Then

(1) $\psi=\bigwedge\{\cdot \otimes(\check{A}, \check{B}):[A, B] \in \mathbf{B}\}$.

(2) Furthermore, if $\mathbf{B}\left(\psi^{*}\right)$, the basis of its dual, satisfies the representation condition for $\psi^{*}$, then

$$
\mathbf{B}\left(\psi^{*}\right) \subset \mathbf{B}, \psi=\bigwedge\left\{\cdot\left(\nabla(\check{A}, \check{B}):[A, B] \in \mathbf{B}\left(\psi^{*}\right)\right\}\right.
$$

and $\psi$ is said to have a minimal representation by an infimum.

Proof. The result of part (1) follows from Theorem 4.1 by applying the inverse of the dual lattice-isomorphism $\psi \mapsto \psi^{*}$ as in the proof of Theorem 2.2. The result of part (2) follows by the same arguments of the proof of part (2) of Theorem 4.1.

We now specialize the minimal representation to the cases of inf-separable, sup-separable, increasing, and decreasing t.i. mappings.

Corollary 4.1. Let $\psi$ be a t.i. mapping. Let $\mathscr{B}(\psi)$, and let $\tilde{\mathscr{B}}(\psi)$ be the sets of the minimal and maximal elements of $\mathscr{K}(\psi)$, respectively. Let $\mathscr{B}\left(\psi^{*}\right)$ and $\tilde{\mathscr{B}}\left(\psi^{*}\right)$ be the similar sets relatively to $\mathscr{K}\left(\psi^{*}\right)$. When explicitly used in the expressions below, these sets are assumed to satisfy the corresponding condition among the following ones:

for any $X \in \mathscr{K}(\psi)$, there exists $X^{\prime} \in \mathscr{B}(\psi)$ such that $X^{\prime} \subset X$,

for any $X \in \mathscr{K}(\psi)$, there exists $X^{\prime} \in \tilde{\mathscr{B}}(\psi)$ such that $X \subset X^{\prime}$,

for any $X \in \mathscr{K}(\psi)$, there exists $X^{\prime} \in \mathscr{B}\left(\psi^{*}\right)$ such that $X^{\prime} \subset X$, then

for any $X \in \mathscr{K}(\psi)$, there exists $X^{\prime} \in \tilde{\mathscr{B}}\left(\psi^{*}\right)$ such that $X \subset X^{\prime}$;

(1) $\quad \psi=(\bigvee\{\cdot \ominus A: A \in \mathscr{B}(\psi)\}) \wedge\left(\bigvee\left\{\cdot{ }^{c} \ominus B^{c}: B \in \tilde{B}(\psi)\right\}\right)$ if $\psi$ is inf-separable;

(2) $\quad \psi=\left(\bigwedge\left\{\cdot \oplus \check{A}: A \in \tilde{\mathscr{B}}\left(\psi^{*}\right)\right\}\right) \vee\left(\bigwedge\left\{\cdot^{c} \oplus \check{B}^{c}: B \in \tilde{\mathscr{B}}\left(\psi^{*}\right)\right\}\right)$ if $\psi$ is sup-separable;

(3) $\quad \psi=\bigvee\{\cdot \ominus A: A \in \mathscr{B}(\psi)\}=\bigwedge\left\{\cdot \oplus \check{A}: A \in \mathscr{B}\left(\psi^{*}\right)\right\}$ if $\psi$ is increasing;

(4) $\quad \psi=\bigvee\left\{\cdot{ }^{c} \ominus B^{c}: B \in \tilde{\mathscr{B}}(\psi)\right\}=\bigwedge\left\{\cdot{ }^{c} \oplus \check{\boldsymbol{B}}^{c}: \tilde{B} \in \tilde{\mathscr{B}}\left(\psi^{*}\right)\right\}$ if $\psi$ is decreasing. 
Proof. (1) The inf-separable case. Because of Property 3.1, the condition $(X \in$ $\mathscr{B}(\psi), Y \in \tilde{\mathscr{B}}(\psi)$, and $X \subset Y)$ is equivalent to $[X, Y] \in \mathbf{B}(\psi)$. The result then follows by using Theorem 4.1 since under the assumption on $\mathscr{B}(\psi)$ and $\mathscr{B}(\psi), \mathbf{B}(\psi)$ satisfies the representation condition.

(2) The sup-separable case. The result follows by using Theorem 4.2 and the arguments of part (1) since $\psi^{*}$ is inf-separable.

(3) The increasing case. The results follow by using the arguments of the proof of Theorems 4.1 and 4.2 and by using Corollary 3.1. Actually the results cannot be derived directly from Theorem 4.1 (respectively, Theorem 4.2) because the assumption on $\mathscr{B}(\psi)$ (respectively, $\mathscr{B}\left(\psi^{*}\right)$ ) is not sufficient to guarantee that $\mathbf{B}(\psi)$ (respectively, $\mathbf{B}\left(\psi^{*}\right)$ ) satisfies the representation condition for $\psi$ (respectively, $\psi^{*}$ ).

(4) The decreasing case. The result follows by similar arguments.

In his thesis, Maragos (1985) gives a sufficient condition on an increasing t.i. mapping $\psi$ from $\mathscr{F}$ (the collection of closed subsets of a topological space $E$ ) to $\mathscr{P}(E)$ under which $\mathscr{R}(\psi)$ satisfies the corresponding condition given in Corollary 4.1.

4.2. Topological aspects. In this section, we give a sufficient condition on a t.i. mapping $\psi$, the domain of which is the collection of closed subsets of a topological space, under which $\mathbf{B}(\psi)$ satisfies the representation condition for $\psi$. Actually, this condition appears to be the same as for the increasing case of Maragos.

For the moment, let $E$ represent a given topological space which is assumed to be locally compact (i.e., each point in $E$ admits a compact neighborhood), Hausdorff, and separable (i.e., the topology of $E$ admits a countable base). Let $\mathscr{F}$ be the collection of closed subsets of $E$. The collection $\mathscr{F}$ is assumed to be topologized in the way proposed by Matheron (1975). Following Matheron, the selected topology on $\mathscr{F}$ is the one generated by the set of collections of the type

$$
\mathscr{F}^{K}=\{X \in \mathscr{F}: X \cap K=\varnothing\},
$$

where $K$ is a compact subset of $E$, and

$$
\mathscr{F}_{G}=\{X \in \mathscr{F}: X \cap G \neq \varnothing\},
$$

where $G$ is an open subset of $E$. topology.

As in Serra (1982) and Maragos (1985), we call this topology the hit-miss

The set of the collections of the type

$$
\mathscr{F}^{K}
$$

or

$$
\mathscr{F}_{G_{1}} \cap \cdots \cap \mathscr{F}_{G_{n}} \quad(n \geqq 1)
$$

or

$$
\mathscr{F}^{K} \cap \mathscr{F}_{G_{1}} \cap \cdots \cap \mathscr{F}_{G_{n}} \quad(n \geqq 1)
$$

is a base for the hit-miss topology.

The open sets in this base are collections of closed sets of $E$ that miss a compact set of $E$ or that hit $n$ open sets of $E$ or, again, that miss a compact set of $E$ and hit $n$ open sets of $E$. 
LeMMA 4.1. Let $\mathscr{L}$ be a subset of $\mathscr{F}$, linearly ordered (under set inclusion); then $\cap \mathscr{L}$ and $\bar{U}$ are adherent points of $\mathscr{L}$ in $\mathscr{F}$ (i.e., with respect to the hit-miss topology), that is,

\section{$\cap \mathscr{L}$ and $\overline{\cup \mathscr{L}} \in \overline{\mathscr{L}}$ in $\mathscr{F}$.}

Proof. Let $M=\cap \mathscr{L}$ or $\bar{U}$. It is sufficient to show that for any open set $\mathscr{A}$ of the type defined by (4.1) such that $M \in \mathscr{A}, \mathscr{A} \cap \mathscr{L} \neq \varnothing$. In other words, for any integer $n$ and any $G_{1}, \cdots, G_{n}$ (open sets of $E$ ), and any $K$ (compact set of $E$ ) such that $M \cap G_{i} \neq \varnothing(i=1, \cdots, n)$ and $M \cap K=\varnothing$, it has to be proved that there exists $X \in \mathscr{L}$ such that $X \cap G_{i} \neq \varnothing(i=1, \cdots, n)$ and $X \cap K=\varnothing$.

(1) The case of $M=\cap \mathscr{L}$. First, for any $X \in \mathscr{L}, M \subset X$, therefore, for any integer $n$ and any open set of $E, G_{i}(i=1, \cdots, n)$, such that $M \cap G_{i} \neq \varnothing$, we have $X \cap G_{i} \neq \varnothing$ since $\varnothing \neq M \cap G_{i} \subset X \cap G_{i}(i=1, \cdots, n)$; second, let $K$ be any compact set of $E$ such that $M \cap K=\varnothing$, that is, such that $K \subset M^{c}$. The set $A=M^{c}$ is an open set and can be written as $A=\bigcup \mathcal{M}$, where $\mathcal{M}=\left\{Y \subset E: Y^{c} \in \mathscr{L}\right\}$. The collection $\mathcal{M}$ is linearly ordered and is an open covering of $K$. The set $K$ being a compact set of $E$, we have that there exists a finite subcovering of $K$, say $\mathcal{M}^{\prime}$. The collection $\mathcal{M}^{\prime}$ being linearly ordered and finite, we have $\cup \mathcal{M}^{\prime} \in \mathcal{M}$. Therefore, there exists $Y \in \mathcal{M}$ (namely, $Y=\cup \mathcal{M}^{\prime}$ ) such that $K \subset Y \subset A$ or, equivalently, there exists $X \in \mathscr{L}$ (namely, $X=Y^{c}$ ) such that $K \cap X=\varnothing$.

(2) The case of $M=\overline{\cup \mathscr{L}}$. First, for any $X \in \mathscr{L}, X \subset M$, therefore, for any compact set of $E, K$, such that $M \cap K=\varnothing$, we have $X \cap K=\varnothing$, since $X \cap K \subset M \cap K=\varnothing$; second, for any integer $n$ and any open set of $E, G_{i}(i=1, \cdots, n)$, such that $M \cap G_{i} \neq \varnothing$, by closure property, $(\cup \mathscr{L}) \cap G_{i} \neq \varnothing$. Let $x_{i} \in(\cup \mathscr{L}) \cap G_{i}$; by definition of $\cup \mathscr{L}$, there exists $X_{i} \in \mathscr{L}$ such that $x_{i} \in X_{i}$. In other words, there exists $X_{i} \in \mathscr{L}$ such that $X_{i} \cap G_{i} \neq \varnothing$. Let $\mathscr{L}^{\prime}$ be the collection of the $X_{i}(i=1, \cdots, n)$. The collection $\mathscr{L}^{\prime}$ being linearly ordered and finite, we have $\cup \mathscr{L}^{\prime} \in \mathscr{L}$. Let $X=\cup \mathscr{L}^{\prime}, X_{i} \subset X(i=1, \cdots, n)$, which proves that there exists $X \in \mathscr{L}$ such that $X \cap G_{i} \neq \varnothing(i=1, \cdots, n)$.

LEMMA 4.2. Let $\left\{A_{i}: i \in \mathbb{N}\right\}$ and $\left\{B_{i}: i \in \mathbb{N}\right\}$ be two sequences in $\mathscr{F}$ such that $A_{i} \subset$ $B_{i}(i \in \mathbb{N}), A_{i} \downarrow A$ and $B_{i} \uparrow B$ in $\mathscr{F}$, and let $X \in \mathscr{F}$ be such that $A \subset X \subset B$. Then there exists a sequence $\left\{X_{i}: i \in \mathbb{N}\right\}$ in $\mathscr{F}$ such that $A_{i} \subset X_{i} \subset B_{i}(i \in \mathbb{N})$ and $\lim X_{i}=X$ in $\mathscr{F}$.

Proof. Let $X_{i}=\left(A_{i} \cup X\right) \cap B_{i}(i \in \mathbb{N})$; then, for any $i \in \mathbb{N}, X_{i} \in \mathscr{F}, X_{i} \subset B_{i}$, and $A_{i} \subset$ $X_{i}$. This last inclusion is true since, by distributivity, $X_{i}=\left(A_{i} \cap B_{i}\right) \cup\left(X \cap B_{i}\right)$, because $A_{i} \subset B_{i}, X_{i}=A_{i} \cup\left(X \cap B_{i}\right)$. By Corollary 3.d of Matheron $\left(1975\right.$, p. 7) (with $F_{n}=X$ and $F_{n}^{\prime}=B_{n}$ ),

$$
\lim \left(X \cap B_{i}\right)=\overline{X \cap B} \text { in } \mathscr{F} \text {. }
$$

By Corollary 3.a of Matheron (1975, p. 7),

$$
\lim A_{i}=A \text { in } \mathscr{F} \text {. }
$$

By Corollary 1 of Matheron $(1975$, p. 7), on continuity of the union,

$$
\lim \left(A_{i} \cup\left(X \cap B_{i}\right)\right)=\left(\lim A_{i}\right) \cup\left(\lim \left(X \cap B_{i}\right)\right) \text { in } \mathscr{F}
$$

In other words, from the above three equalities on limits,

$$
\lim X_{i}=A \cup(\overline{X \cap B}) \text { in } \mathscr{F} \text {. }
$$

By assumption, $A \subset X \subset B$ and $X \in \mathscr{F}$; therefore,

$$
A \cup(\overline{X \cap B})=A \cup \bar{X}=A \cup X=X .
$$

This proves that there exists $\left\{X_{i}: i \in \mathbb{N}\right\}$ in $\mathscr{F}$ such that $A_{i} \subset X_{i} \subset B_{i}(i \in \mathbb{N})$ and $\lim X_{i}=$ $X$. 
With respect to the inclusion, the collection $\mathscr{F}$ of closed subsets of $E$ is a complete lattice and the set of the closed intervals of $\mathscr{F}$ is a complete joint-semilattice.

Lemma 4.3. Let $\mathscr{C} \subset \mathscr{F}$, and let $\mathbf{C}$ be a linearly ordered (under set inclusion) set of closed intervals of $\mathscr{F}$ contained in $\mathscr{C}$; then

$$
\text { Sup } \mathbf{C} \subset \overline{\mathscr{C}} \quad \text { in } \mathscr{F} \text {. }
$$

Proof. Let $\mathscr{L}$ be the collection of the extremities of the closed intervals in $\mathbf{C}$ :

$$
\mathscr{L}=\left\{X \in \mathscr{F}: \exists\left[X, X^{\prime}\right] \text { or }\left[X^{\prime}, X\right] \in \mathbf{C}\right\} .
$$

$\mathscr{L}$ inherits the linearly ordered property of $\mathbf{C}$; therefore, by Lemma $4.1, \cap \mathscr{L}$ and $\bar{U} \mathscr{L} \in \overline{\mathscr{L}}$ in $\mathscr{F}$. It is known (Matheron (1975, Thm. 1.2.1)) that the hit-miss topology is separable; therefore (Dugundji (1966, Thm. 6.2, p. 218)) there exist two sequences $\left\{A_{i}, i \in \mathbb{N}\right\}$ and $\left\{B_{i}, i \in \mathbb{N}\right\}$ in $\mathscr{L}$ such that $\lim A_{i}=\cap \mathscr{L}$ and $\lim B_{i}=\bar{U}$ in $\mathscr{F}$. These sequences can be chosen, respectively, decreasing and increasing and such that $A_{i} \subset$ $B_{i}(i \in \mathbb{N})$. By Corollary 3.a-b of Matheron (1975, p. 7),

$$
\lim A_{i}=\cap\left\{A_{i}, i \in \mathbb{N}\right\} \text { in } \mathscr{F}
$$

and

$$
\lim B_{i}=\overline{U\left\{B_{i}, i \in \mathbb{N}\right\}} \text { in } \mathscr{F} \text {. }
$$

In other words, under the linearly ordered assumption, there exist two sequences $\left\{A_{i}, i \in \mathbb{N}\right\}$ and $\left\{B_{i}, i \in \mathbb{N}\right\}$ in $\mathscr{L}$ such that $A_{i} \subset B_{i}(i \in \mathbb{N}), A_{i} \downarrow \cap \mathscr{L}$ and $B_{i} \uparrow \cup \mathscr{L}$. Let $X \in \operatorname{Sup} \mathbf{C}$, the infimum and supremum of $\mathscr{L}$ in $\mathscr{F}$ being, respectively, $\cap \mathscr{L}$ and $\bar{U}$, we have $\cap \mathscr{L} \subset X \subset \overline{U \mathscr{L}}$. By Lemma 4.2 (with $A=\cap \mathscr{L}$ and $B=\bar{\cup} \mathscr{L}$ ), there exists a sequence $\left\{X_{i}, i \in \mathbb{N}\right\}$ in $\mathscr{F}$ such that $A_{i} \subset X_{i} \subset B_{i}(i \in \mathbb{N})$, that is, $X_{i} \in\left[A_{i}, B_{i}\right](i \in \mathbb{N})$, and $\lim X_{i}=X$ in $\mathscr{F}$. Because of the way $\mathscr{L}$ has been constructed from $\mathbf{C}$, there exists a closed interval in $\mathbf{C}$ which contains $\left[A_{i}, B_{i}\right]$; therefore, the $X_{i}$ 's are in $\mathscr{C}$ by definition of $C$. This means that $X$ is an adherent point of $\mathscr{C}$ in $\mathscr{F}$ (Dugundji (1966, Thm. 6.2, p. 218)), that is, $X \in \overline{\mathscr{C}}$ in $\mathscr{F}$.

From now on, $E$ is the $d$-dimensional Euclidean space $\mathbb{R}^{d}$ or its subset $\mathbb{Z}^{d}$, equipped, respectively, with the Euclidean topology or with the relative Euclidean topology, and the t.i. mappings under consideration are from $\mathscr{F}$, the set of closed subsets of $E$ ( $\mathscr{F}$ is closed under translation), to $\mathscr{P}(E)$. It can be observed that the Euclidean topology or the relative Euclidean topology in $\mathscr{F}$ satisfy all the assumptions made on the topological space $E$ at the beginning of this subsection (Maragos (1985)).

A mapping $\psi$ from $\mathscr{F}$ to $\mathscr{F}$ is upper semicontinuous (u.s.c.) if and only if for any compact subset $K$ of $E$, the set $\psi^{-1}\left(\mathscr{F}^{K}\right)$ is open in $\mathscr{F}$ (Matheron $(1975$, p. 8$)$ ).

THEOREM 4.3 (Property of the basis of an u.s.c. t.i. mapping). Let $\psi$ be an u.s.c. t.i. mapping from $\mathscr{F}$ to $\mathscr{F} ;$ then $\mathbf{B}(\psi)$, its basis, satisfies the representation condition for $\psi$.

Proof (the logic of this proof is similar to that of the proof of Theorem 5.7 in Maragos (1985)). Let $[A, B]$ be a closed interval contained in $\mathscr{K}(\psi)$; it is always possible to construct a linearly ordered set $\mathbf{L}$ of closed intervals contained in $\mathscr{K}(\psi)$ such that $[A, B] \in \mathbf{L}$. By Lemma 2.1 of Maragos (1985), there exists a maximal linearly ordered set $\mathbf{M}$ of closed intervals contained in $\mathscr{K}(\psi)$ such that $\mathbf{L} \subset \mathbf{M}$. Therefore, there exists a closed interval $\left[A^{\prime}, B^{\prime}\right]$, namely, $\left[A^{\prime}, B^{\prime}\right]=\operatorname{Sup} \mathbf{M}$, which contains $[A, B]$ :

$$
[A, B] \subset \operatorname{Sup} \mathbf{L} \subset \operatorname{Sup} \mathbf{M}=\left[A^{\prime}, B^{\prime}\right] .
$$

By Proposition 8.2.1 of Matheron (1975), $\mathscr{K}(\psi)$ is closed in $\mathscr{F}$, that is, $\overline{\mathscr{K}(\psi)}=\mathscr{K}(\psi)$. By using Lemma 4.3 (with $\mathscr{C}=\mathscr{K}(\psi)$ and $\mathbf{C}=\mathbf{M}$ ), $\left[\boldsymbol{A}^{\prime}, B^{\prime}\right] \subset \mathscr{K}(\psi)$. Furthermore, $\mathbf{M}$ being a maximal set of closed intervals contained in $\mathscr{K}(\psi)$, we have $\left[A^{\prime}, B^{\prime}\right] \in \mathbf{B}(\psi)$, 
because otherwise there would exist another closed interval contained in $\mathscr{K}(\psi)$ that contains $\left[A^{\prime}, B^{\prime}\right]$. In other words, there would exist a linearly ordered set of closed intervals contained in $\mathscr{K}(\psi)$ that properly contains $\mathbf{M}$ and $\mathbf{M}$ would not be maximal.

In terms of minimal representation, Theorem 4.3 leads to the following result.

Let $\mathscr{G}$ be the collection of open subsets of $E$.

COROLlARY 4.2. (1) If $\psi$ is an u.s.c. t.i. mapping from $\mathscr{F}$ to $\mathscr{F}$, then $\psi$ has a minimal representation by a supremum.

(2) If $\psi$ is a t.i. mapping from $\mathscr{G}$ to $\mathscr{G}$, which has an u.s.c. dual $\psi^{*}$ from $\mathscr{F}$ to $\mathscr{F}$, then $\psi$ has a minimal representation by an infimum.

Proof. (1) The result in part (1) follows by using Theorems 4.1 and 4.3.

(2) If $\psi^{*}$ is u.s.c. then, by Theorem $4.3, \mathrm{~B}\left(\psi^{*}\right)$ satisfies the representation condition for $\psi^{*}$. Hence the result in part (2) follows by using Theorem 4.2.

When $E=\mathbb{Z}^{d}$ is equipped with the relative Euclidean topology, then $\mathscr{F}=\mathscr{G}=\mathscr{P}(E)$ and parts (1) and (2) of Corollary 4.2 may be relative to the same t.i. mapping.

Before ending this section, let us say some words about the representation by a supremum for the sup-generating mapping $\bullet(A, B)$. By using Corollary 4 of Matheron $(1975$, p. 7$)$, any closed interval $[A, B]$ of $\mathscr{F}$ is closed in $\mathscr{F}$. Since $\mathscr{K}(\cdot \otimes(A, B))=[A, B]$, by Proposition 8.2.1 of Matheron (1975), the sup-generating mapping $\bullet(A, B)$ is u.s.c. Therefore, by Theorem 4.3. its basis satisfies the representation condition for - $(1)(A, B)$. Actually, its basis is the singleton $\{[A, B]\}$. In the next section more examples are presented.

5. Examples. We now give some simple examples to illustrate the theory. In this section, $E$ is the d-dimensional Euclidean space $\mathbb{R}^{d}$ or its subset $\mathbb{Z}^{d}$, equipped, if necessary, respectively, with the Euclidean topology or with the relative Euclidean topology. Finally, $\mathscr{F}$ and $\mathscr{G}$ are, respectively, the collections of closed and open subsets of $E$.

5.1. Complementary mapping. In this section we will study the representation by a supremum for the mapping ${ }^{c}$ which associate the complementary set $X^{c}$ to a set $X$ in $\mathscr{A} \subset \mathscr{P}(E) .{ }^{c}$ is an example of decreasing t.i. mapping.

The kernel of $\cdot^{c}$ is

$$
\mathscr{K}\left(\cdot \cdot^{c}\right)=\{X \in \mathscr{A}: \mathbf{o} \notin X\} .
$$

If $E-\{$ o $\} \in \mathscr{A}$, then the collection $\tilde{\mathscr{B}}\left(\cdot^{c}\right)$ of maximal elements of $\mathscr{K}\left(\cdot^{c}\right)$ is the singleton $\{E-\{0\}\}$. This collection satisfies the condition of Corollary 4.1 which leads to the formula

$$
\cdot^{c}={ }^{c} \ominus\{\mathbf{o}\} .
$$

If $\varnothing$ and $E-\{o\} \in \mathscr{A}$, then $\mathscr{K}\left(\cdot^{c}\right)$ is simply the closed interval $[\varnothing,(E-\{\mathrm{o}\})]$ and $\mathbf{B}\left(\cdot^{c}\right)$, the basis of $\cdot^{c}$, is the singleton $\{[\varnothing, E-\{0\}]\}$. This basis satisfies the representation condition for ${ }^{c}$ and Theorem 4.1 leads to the formula

$$
\cdot^{c}=\cdot \otimes(\varnothing, E-\{\mathbf{o}\}) \text {. }
$$

If $E=\mathbb{R}^{d}$ and $\mathscr{A}=\mathscr{F}$, then $E-\{\mathrm{o}\}$ is an open set, that is, it does not belong to the domain of $\cdot^{c}$ and the above simplifications do not occur. In this case, there is no maximal closed interval contained in $\mathscr{K}\left(\cdot^{c}\right)$ (Banon and Barrera, $(1990)$ ), $\mathbf{B}\left(\cdot^{c}\right)$ is empty and it does not satisfy the representation condition for $\cdot^{c}$. The mapping ${ }^{c}$ has no minimal representation by a supremum. The only simplification that works comes from the decreasing property, and by using it, Corollary 3.1 leads to the formula

$$
\cdot^{c}=\bigvee\left\{\cdot{ }^{c} \ominus B: o \in B \in \mathscr{G}\right\} \text {. }
$$


If $E=\mathbb{Z}^{d}$ and $\mathscr{A}=\mathscr{F}$, then $\mathscr{A}=\mathscr{P}(E), \varnothing$ and $E-\{\mathrm{o}\} \in \mathscr{A}$, and the above analysis, leading to a minimal representation, holds. By Corollary 4 of Matheron $(1975$, p. 7$)$,

$$
\mathscr{K}\left(\cdot{ }^{c}\right)=\{X \in \mathscr{F}: X \subset E-\{\mathbf{o}\}\}
$$

is closed in $\mathscr{F}$ and, by Property 8.2.1 of Matheron (1975),.$^{c}$ is u.s.c. from $\mathscr{F}$ to $\mathscr{F}$. Therefore, by Corollary 4.2 , we have a confirmation that $\cdot^{c}$ has a minimal representation by a supremum.

5.2. Edge extraction. In this section we study the representation by a supremum for an inf-separable mapping, which is useful in extracting the edges of an image.

Let $D \in \mathscr{P}(E)$ ( $D$ with more than one element). The edge extraction mapping we consider is given by

$$
\psi=(\cdot \oplus D) \cap\left(\cdot{ }^{c} \oplus D\right) ;
$$

in other words,

$$
\psi(X)=(X \oplus D)-(X \ominus D) \quad(X \in \mathscr{A}) .
$$

By construction, $\psi$ is an inf-separable mapping and its kernel is

$$
\mathscr{K}(\psi)=\left\{X \in \mathscr{A}: X \cap \check{D} \neq \varnothing \text { and } X^{c} \cap \check{D} \neq \varnothing\right\} .
$$

Figure 5.1 shows two sets $A$ and $B$ that belong to $\mathscr{K}(\psi)$.

If $\mathscr{A}=\mathscr{P}(E)$, then the collections $\mathscr{R}(\psi)$ and $\tilde{\mathscr{B}}(\psi)$ of Corollary 4.1 are

$$
\begin{aligned}
& \mathscr{B}(\psi)=\{X \in \mathscr{A}: X=\{x\} \text { and } x \in \check{D}\}, \\
& \tilde{\mathscr{B}}(\psi)=\left\{X \in \mathscr{A}: X=\{x\}^{c} \text { and } x \in \check{D}\right\} .
\end{aligned}
$$

These collections satisfy the conditions of Corollary 4.1, which leads to the formula

$$
(\cdot \oplus D) \cap\left(\cdot{ }^{c} \oplus D\right)=(\bigvee\{\cdot \ominus\{x\}: x \in \check{D}\}) \wedge\left(\bigvee\left\{\cdot{ }^{c} \ominus\{x\}: x \in \check{D}\right\}\right)
$$

If $E=\mathbb{Z}^{d}$ and $\mathscr{A}=\mathscr{F}$, then $\mathscr{A}=\mathscr{P}(E)$ and $\psi$ is continuous, as the intersection of two continuous mappings, that is, $\psi$ is, in particular, u.s.c. from $\mathscr{F}$ to $\mathscr{F}$. Therefore, by Corollary 4.2 we have a confirmation that the above edge extraction mapping $\psi$ has a minimal representation by a supremum.

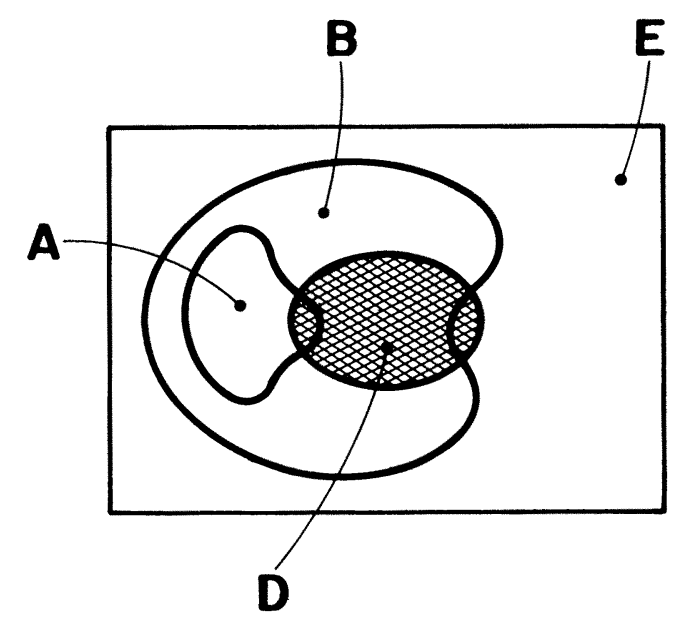

FIG. 5.1. Example of two sets $A$ and $B$ belonging to the kernel $\mathscr{K}\left((\cdot \oplus D) \cap\left({ }^{c} \oplus D\right)\right)$ of an edge detection mapping characterized by $D$. A and $B^{c}$ must hit $D$. 
5.3. Representation by an infimum for a sup-generating mapping. In this section we study the representation by an infimum for the sup-generating mapping $\psi=\cdot \otimes(A, B)(A \subset B)$ of $\S 2.1$.

Let $\mathscr{A}$ be the domain of $\psi$. The dual mapping of $\psi$ is the inf-generating mapping $\psi^{*}=\cdot \otimes(\check{A}, \check{B})$ of $\S 2.2$ and its kernel is

$$
\mathscr{K}\left(\psi^{*}\right)=\left\{X \in \mathscr{A}^{*}: X \cap \boldsymbol{A} \neq \varnothing \text { or } \boldsymbol{X} \cup B \neq E\right\} .
$$

Figure 5.2 shows the extremities $U$ and $V$ of two types of closed intervals contained in $\mathscr{K}\left(\psi^{*}\right)$.

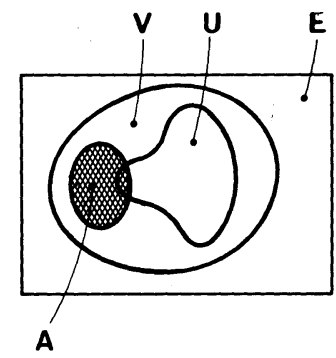

(a)

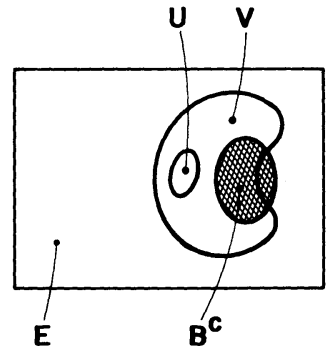

(b)

FIG. 5.2. Example of extremities $U$ and $V$ of two types of closed intervals $[U, V]$ contained in the kernel $\mathscr{K}\left((\cdot \otimes(A, B))^{*}\right)$ of the dual of a sup-generating mapping characterized by $A$ and $B$. (a) $U$ must hit $A$, or (b) $V$ must not contain $B^{c}$.

If $\mathscr{A}=\mathscr{P}(E)$, then $\mathscr{A}^{*}=\mathscr{A}, \mathscr{K}\left(\psi^{*}\right)$ is nonempty whenever $\boldsymbol{A} \neq \varnothing$ or $\boldsymbol{B} \neq E$ and the basis of $\psi^{*}$ is the set of closed intervals of type $[\{x\}, E]$ with $x \in A$ or $\left[\varnothing,\{x\}^{c}\right]$ with $x \in B^{c}$. This basis satisfies the representation condition for $\psi^{*}$, hence, Theorem 4.2 leads to the formula

$$
\cdot \otimes(A, B)=(\bigwedge\{\cdot \otimes(\{x\}, E): x \in A\}) \wedge\left(\bigwedge\left\{\cdot \otimes\left(\varnothing,\{x\}^{c}\right): x \in B^{c}\right\}\right)
$$

or simply

$$
\cdot \otimes(A, B)=(\bigwedge\{\cdot \oplus\{x\}: x \in A\}) \wedge\left(\bigwedge\left\{\cdot^{c} \oplus\{x\}: x \in B^{c}\right\}\right) .
$$

If $E=\mathbb{Z}^{d}$ and $\mathscr{A}=\mathscr{G}$, then $\mathscr{A}=\mathscr{F}=\mathscr{P}(E)$ and $\psi^{*}$ is continuous as the union of continuous mappings, that is, $\psi^{*}$ is, in particular, u.s.c. from $\mathscr{F}$ to $\mathscr{F}$. Therefore, by Corollary 4.2 we have a confirmation that the sup-generating mapping $\bullet \otimes(A, B)$ has a minimal representation by an infimum.

Of course, the above formula can be derived in another way, by using the fact that $\bullet(A, B)$ is inf-separable and by applying Corollary 4.1 to its increasing and decreasing parts, that is, to $\ominus \ominus A$ and ${ }^{c} \ominus B^{c}$.

5.4. Shape recognition. As a last example, we study the representation by a supremum of the so-called window transformation, introduced by Crimmins and Brown (1985) in the field of automatic shape recognition.

Let $W \in \mathscr{P}(E)$; a mapping $\psi$ from $\mathscr{A}$ to $\mathscr{P}(E)$ is called a window transformation with respect to the window $W$, if and only if there exists a subcollection $\mathscr{D} \subset \mathscr{P}(W)$ such that

$$
\psi(X)=\left\{x \in E: W \cap X_{-x} \in \mathscr{D}\right\} \quad(X \in \mathscr{A})
$$


The mapping $\psi$ "recognizes" in particular all the shapes in $\mathscr{A}$ which are in $\mathscr{D}$ by producing a point marker. The mapping $\psi$ is t.i. and its kernel is

$$
\mathscr{K}(\psi)=\{X \in \mathscr{A}: W \cap X \in \mathscr{D}\} .
$$

Figure 5.3(a) shows one typical element of $\mathscr{K}(\psi)$ when $W$ is a rectangular and $\mathscr{D}$ contains a triangle.

It is known (Crimmins and Brown (1985); Maragos (1985) that $\psi$ can be written in the following way (using our notation):

$$
\psi=\bigvee\left\{\cdot \otimes\left(U,(W-U)^{c}\right): U \in \mathscr{D}\right\} .
$$

In what follows, in the nontrivial case for which $\mathscr{A} \cap \mathscr{D}$ has more than one element and under some circumstances given below, it is shown that (5.1) is exactly the minimal representation by a supremum for $\psi$.

For the moment, let us assume that the above collection $\mathscr{D}$ satisfies the following assumption.

Assumption 5.1. For any $U_{1}$ and $U_{2} \in \mathscr{D}$, comparable $\left(U_{1} \subset U_{2}\right)$ and distinct $\left(U_{1} \neq U_{2}\right)$, there exists $X \in \mathscr{A}$ such that $U_{1} \subset X \cap W \subset U_{2}$ and $X \cap W \notin \mathscr{D}$.

Under this assumption, the extremities $A$ and $B$ of a closed interval $[A, B]$ contained in $\mathscr{K}(\psi)$ satisfy $A \cap W=B \cap W$.

If $(W-U)^{c} \in \mathscr{A}$ for any $U \in \mathscr{A} \cap \mathscr{D}$, then the maximal closed intervals contained in $\mathscr{K}(\psi)$ are of type $\left[U,(W-U)^{c}\right]$ with $U \in \mathscr{A} \cap \mathscr{D}$. Figures 5.3(b) and 5.3(c) show the extremities of a typical maximal closed interval contained in $\mathscr{K}(\psi)$. The basis of $\psi$, the set of these maximal closed interval, satisfies the representation condition for $\psi$, hence, by using Theorem 4.1 and by noting that $\otimes\left(U,(W-U)^{c}\right)$ is the least t.i. mapping whenever $U \in \mathscr{D}-\mathscr{A}$, we observe that (5.1) is exactly the minimal representation by a supremum for $\psi$.

If $\mathscr{D} \subset \mathscr{F}, \mathscr{D}$ finite, $\mathscr{A}=\mathscr{F}$ and the window $W$ is an open subset of $E$, it can be shown that $\psi$ is an u.s.c. mapping from $\mathscr{F}$ to $\mathscr{F}$ (Banon and Barrera (1990)). Therefore,

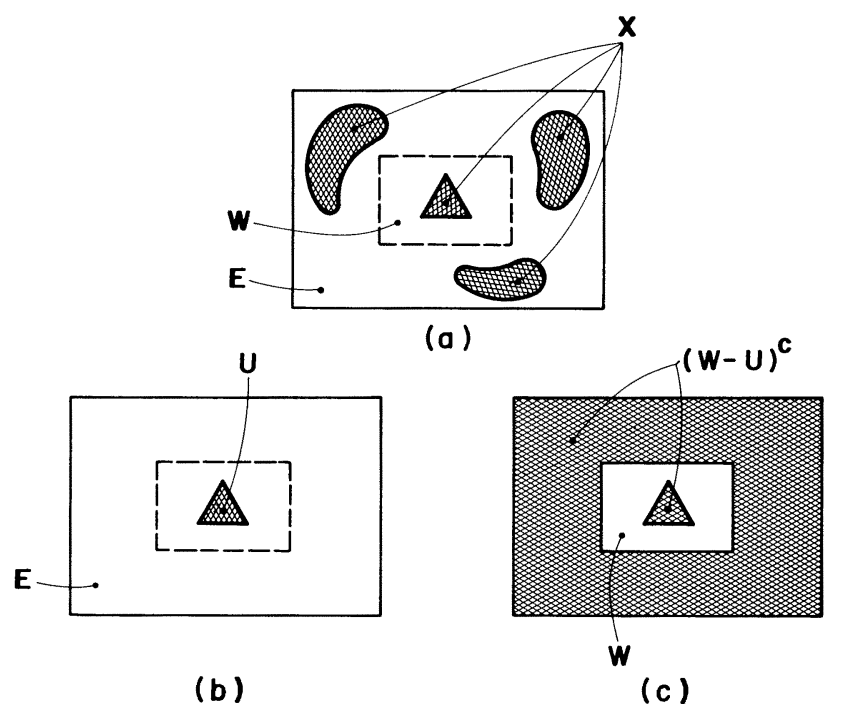

FIG. 5.3. Example of kernel elements of a window transformation with respect to the window $W$ and the collection $\mathscr{D}$ containing at least a triangle $U$. (a) shows a particular element $X(X \cap W=U)$, (b) and (c) show the extremities of the maximal closed interval $\left[U,(W-U)^{c}\right]$ contained in the kernel and relative to the triangle $U$. 
by Corollary 4.1 we have a confirmation that $\psi$ has a minimal representation by a supremum.

If $\mathscr{D}$ does not satisfy Assumption 5.1, then the minimal representation by a supremum may be even simpler than (5.1). In the increasing case, Example 5.9 of Maragos (1985) illustrates this point. In the not necessarily increasing case, the $K$-tolerance matching is another illustrative example.

Let $K$ and $W \in \mathscr{P}(E), K=\check{K}$; a mapping $\psi$ from $\mathscr{A}$ to $\mathscr{P}(E)$ is called $K$-tolerance matching (Haralick (1988)), if and only if there exists a subcollection $\mathscr{T} \subset \mathscr{P}(W)$ such that $\psi$ is a window transformation from $\mathscr{A}$ to $\mathscr{P}(E)$ with respect to $W$ and the subcollection $\mathscr{D}$ defined by

$$
\mathscr{D}=\{X \in \mathscr{P}(E): T \ominus K \subset X \subset(T \oplus K) \cap W \text { and } T \in \mathscr{T}\} .
$$

The mapping $\psi$ "recognizes" in particular, all the shapes in $\mathscr{A}$ that are similar to the ones in $\mathscr{T}$ within $K$-tolerant limits.

As a window transformation, $\psi$ can be represented as in (5.1). On the other hand, $\mathscr{D}$ may not satisfy Assumption 5.1 and a simpler representation may be suspected.

Under an assumption similar to that above, it can be shown that the $K$-tolerance matching $\psi$ can be represented in the following minimal form:

$$
\psi=\bigvee\left\{\cdot \otimes\left(T \ominus K,(W-(T \oplus K))^{c}\right): T \in \mathscr{T}\right\}
$$

(Banon and Barrera (1990)). The representation in (5.2) is simpler than in (5.1) $(\mathscr{T} \subset \mathscr{D})$. Actually, in the general case, the minimal representation by a supremum for a $K$ tolerance matching may even be simpler than (5.2).

6. Conclusion. In this paper, a pair of representations for translation-invariant mappings has been introduced. In $\S 2$, the representation by a supremum has been obtained as an isomorphic property of the decomposition of any subset of a poset as the union of its closed intervals. The representation by an infimum has been obtained by using the duality principle. In $\S 3$, the simplification of the representations for inf-separable and (respectively, sup-separable) mappings have shown that such mappings can be written as the infimum (respectively, supremum) of an increasing and a decreasing mapping. For inf-separable mappings, this property follows by observing that their kernel are convex. In $\S 4$, it has been proved that any upper semicontinuous mapping has a minimal representation by a supremum, that is, the maximal supgenerating mappings used in the represention of $\psi$ are sufficient to represent $\psi$ by a supremum in a minimal way. It is important to note that the upper semicontinuous condition can be applied only for mappings whose domain is the collection of closed subsets of $E$, but that other mappings may have a minimal representation by a supremum. In $\S 5$, some examples of inf-separable mappings and general mappings have been presented. In the case of the window transformations of Crimmins and Brown, the minimal representation by a supremum has given some insight on previously known results.

Finally, three topics for future research can be outlined: the proposed representations are well adapted to be implemented on simple highly parallel architectures, which should lead to efficient image processing; in practice, exact representations may not be necessary; in such a case, it should be possible to construct approximated representations for a mapping from subsets of its basis; the results derived here, for set mappings, should be extended to function mappings, offering a new tool for digital signal processing.

Acknowledgments. The authors are indebted to their colleagues from the morphological group at INPE and, especially, to Dr. Nelson Delfino d'Ávila Mascarenhas and 
Jeblin Antônio Abraão, M.Sc., who have followed all the progress of their work. They are grateful to Marília Prado de Carvalho for her help with English usage.

\section{REFERENCES}

G. J. F. BANON AND J. BARRERA, Study of a pair of dual minimal representations for translation invariant set mappings by mathematical morphology, INPE-5013-RPE/608, São José dos Campos, São Paulo State, Brazil, INPE ("Instituto Nacional de Pesquisas Espaciais”), January 1990.

G. Birkhoff, Lattice Theory, American Mathematical Society, Providence, RI, 1967.

T. R. CRIMMINS AND W. M. BROWN, Image algebra and automatic shape recognition, IEEE Trans. Aerospace Electron. Systems, 21 (1985), pp. 60-69.

E. R. Dougherty And C. R. Giardina, A digital version of the Matheron representation theorem for increasing $\tau$-mapping in terms of a basis for the kernel, in Proc. IEEE Computer Vision and Pattern Recognition, Miami, June 1986.

J. DugundJi, Topology, Allyn and Bacon, Boston, 1966.

C. R. Giardina AND E. R. Dougherty, Morphological methods in image and signal processing, PrenticeHall, Englewood Cliffs, NJ, 1988.

H. HADWIGER, Minkowskische addition und subtraktion beliebiger punktmengen und die theroeme von Erhard Schmidt, Math. Z., 53 (1950), pp. 210-218.

R. M. Haralick, personal communication, 1988.

R. M. HARAlick, S. R. STERnBerg, AND X. ZHUANG, Image analysis using mathematical morphology, IEEE Pattern Analysis and Machine Intelligence, 4 (1987), pp. 532-550.

P. Hill and G. R. Peterson, Introduction to Switching Theory and Logical Design, John Wiley, New York, 1974.

P. A. Maragos, A unified theory of translation-invariant systems with applications to morphological analysis and coding of images, Ph.D dissertation, School of Electronic Engineering, Georgia Institute of Technology, Atlanta, GA, July 1985.

- A representation theory for morphological image and signal processing, IEEE Pattern Analysis and Machine Intelligence, 11 (1989), pp. 586-599.

G. Matheron, Random Sets and Integral Geometry, John Wiley, New York, 1975. , personal communication, 1989.

H. Minkowski, Volumen und oberfläche, Math. Ann., 57 (1903), pp. 447-495.

J. SerRA, Image Analysis and Mathematical Morphology, Academic Press, New York, 1982.

- Thickenings, thinnings, Tech. Report N-39, Centre de Morphologie Mathématique, École des Mines de Paris, Fontainebleau, France, June 1987, p. 27.

S. R. STER NBERG, Cellular computers and biomedical image processing, in Biomedical Images and Computers, J. Sklansky and J. C. Bisconte, eds., Lecture Notes in Medical Informatics, Springer-Verlag, Berlin, Vol. 17, 1982, pp. 294-319. 